\title{
Chemical Functionalization of Carbon Nano- materials: Bridging the Gap between Simple Carriers and Smart (Metal-free) Catalysts
}

\author{
Giulia Tuciab, Lapo Luconia ${ }^{\text {, Andrea Rossin }}{ }^{a}$, and Giuliano Giambastiani ${ }^{\star a c}$
}

\begin{abstract}
The last few years have witnessed a wonderful technological renaissance that boosted the development of carbon-based nanomaterials (CNMs) doped with light heteroelements and featuring hierarchical porous architectures as valuable metal-free catalysts for a number of key industrial transformations. To date, several approaches to their synthesis have been developed, although many of them lack any real control of the final doping and composition. In contrast, chemical functionalization offers a unique and powerful tool to tailor CNMs' chemical and electronic surface properties as a function of their downstream application in catalysis. Different catalytic processes (hydrolysis/esterification/transesterification reactions, $\mathrm{C}-\mathrm{C}$ bond forming reactions, $\mathrm{CO}_{2}$ derivatization into products of added value and electrochemical oxygen reduction reactions (ORR)) can be conveniently promoted by these materials. In addition, selected examples from this series offer a valuable platform for the in-depth comprehension of the underlying reaction mechanisms. This perspective article offers an overview on the main examples of ad hoc chemically decorated CNMs successfully exploited as metal-free catalysts, highlighting at the same time the importance of the surface chemistry control for the design of more active, metal-free and single-phase heterogeneous catalysts.
\end{abstract}

Keywords: C-C bond formation $\cdot$ Chemical functionalization $\cdot \mathrm{CO}_{2}$ valorization $\cdot$ Metal-free heterogeneous catalysts · ORR

\section{Introduction}

With their unique physical and chemical properties, carbon nanomaterials (CNMs) represent nowadays one of the most valuable tools in heterogeneous catalysis. Beyond their application as simple supports for metal nanoparticles, ${ }^{[1,2]} \mathrm{CNMs}$ as such or in the form of light-heterodoped systems have shown excellent properties as metal-free heterogeneous catalysts in a series of key transformations. The real nature of the active sites responsible of their ultimate catalytic activity is far from being definitively unveiled, as well as their final mechanism of action. However, it is commonly accepted that surface topological defects and electronic redistributions driven by the presence of functional groups play a crucial role on the catalytic performance of these materials. For this reason, the last decade has shown a real boost to-

${ }^{*}$ Correspondence: Dr. G. Giambastiani E-mail: giuliano.giambastiani@iccom.cnr.it alnstitute of Chemistry of OrganoMetallic Compounds ICCOM-CNR, Florence research area

Via Madonna del Piano 10, 50019 Sesto Fiorentino, Florence, Italy

'Dipartimento di Chimica 'Ugo Schiff', Università di

Firenze, 50019 Sesto Fiorentino, Italy

"Kazan Federal University

420008 Kazan, Russian Federation wards the development of carbon nanomaterials, particularly in the form of systems doped with light elements (mainly N, B, P and $\mathrm{S}$ ). The inclusion of hetero-elements is claimed to break the materials' electroneutrality, causing a significant redistribution of the surface electronic charge along with important changes on the final material morphology. ${ }^{[3-5]}$ As a result, doped carbon nanomaterials have been shown to be valuable catalysts offering excellent performance and durability in selected catalytic processes, often with better results than those of the benchmark metal-based catalysts of the state-of-the-art. Many efforts have been devoted to the comprehension of the underpinning mechanisms and nature of the active sites of doped-CNMs engaged in selected catalytic transformations. However, classical 'in situ' synthetic approaches used for their large-scale production (i.e. chemical vapour deposition or post-synthetic thermal treatment of CNMs with heteroatom-containing precursors) are not suitable to achieve a satisfactory control of the nature and degree of the hetero-element doping. Consequently, any unambiguous identification of the 'active sites' engaged in a specific transformation is hard if not totally arbitrary at times. Chemical functionalization represents a valuable alternative to the rational design of hetero-doped CNMs. It allows for a fine tuning of the grafted functionalities and it makes them fully available at the nanoma- terial surface where the catalytic process takes place. ${ }^{[6]}$ To date, many different functionalization strategies for the surface chemical decoration of CNMs have been successfully developed. ${ }^{[7,8]}$ In addition, the unambiguous chemical identity of the surface-grafted functionalities offers valuable clues to debate on the structure/ activity relationships of these systems in catalysis. This perspective focuses on specific examples of metal-free carbon-based materials decorated ad hoc with chemically tethered surface functionalities and applied as heterogeneous catalysts. A fine tuning of the functional groups chemically grafted to 1D-2D carbon nanostructures is highly desired for the following reasons: a) to generate single-phase, solid and stable organocatalysts with excellent performance in selected reactions; b) to show the ability of metal-free systems to carry out processes typically accomplished by metal-based catalysts, including platinum group metals (PGMs); c) to highlight the existence of synergistic effects between the $\mathrm{C}$-nanostructure and its dangling organic groups (non-innocent macromolecular carrier); d) to provide a useful and systematic tool to shed light on the underlying mechanisms of action for these metal-free systems in catalysis. Table 1 lists all the selected catalytic transformations promoted by covalently-decorated carbon nanomaterials as metal-free systems and discussed in sections 2.1-2.4. 


\section{Catalytic Reactions Promoted by ad hoc Functionalized Carbon Nanomaterials as Metal-free Systems}

\subsection{Hydrolysis, Esterification and Transesterification Reactions}

One important application of chemically functionalized CNMs as metal-free catalysts is linked to the exploitation of the acidic or basic character at their outer surface. Feng and co-workers have pioneered this research area with the development of chemically grafted aryl sulfonic acids to the surface of ordered mesoporous carbons. ${ }^{[9]}$ These solid acid catalysts are easy to recycle and show excellent performance coupled with long term durability in biodiesel production. ${ }^{[10]}$ Since these seminal outcomes, the same acidic functionalities covalently linked to the surface of different carbon supports were also tested as heterogeneous catalysts in the hydrolysis of esters and in transesterification reactions. Fan and co-workers reported on the direct grafting of sulfonic acid aryl radicals to reduced graphene oxide (rGO) (Fig. 1).[11] The synthetic approach used in this work was found to prevent undesired sidereactions typically occurring upon direct CNM sulfonation (direct treatment with sulfuric acid). Later, Bitter and co-workers described a related protocol for the chemical decoration of carbon nanofibers with sulfonic acid groups via aryl-diazonium salt chemistry. ${ }^{[12]}$ The as-prepared samples, due to the high accessibility of surface acidic groups, outperformed benchmark catalysts such as Amberlyst-15 and previ-

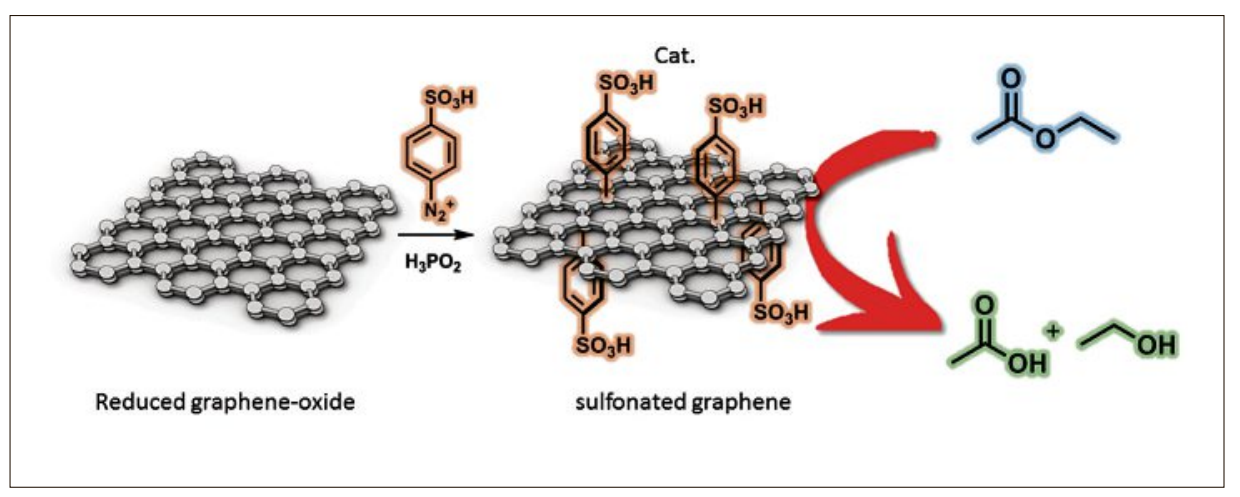

Fig. 1. Functionalization of reduced graphene-oxide (rGO) with aryl-sulfonated groups. Figure loosely reproduced with permission of the Royal Society of Chemistry: Chem. Sci. 2011, 2, 484.

ously reported sulfonated carbons; they also show better stability than acidic carbon materials obtained by direct treatment with sulfuric acid. CNMs decorated with basic functional groups are also excellent candidates as catalytic materials in transesterification reactions. Su and Tessonier reported on the synthesis of decorated CNMs with tertiary aliphatic amines as base catalysts for the transesterification of glyceryl tributyrate with methanol, a model reaction for the production of biodiesel. ${ }^{[13-15]}$ The authors proposed an elegant, clean and fast approach to the chemical grafting of their targeted functionalities, limiting the occurrence of side-reactions at the CNM (potentially detrimental for its performance in the biomass conversion). Their results have clearly outlined the importance of a fine control of the nature of surface functionalities at CNMs. Indeed, longer and less selective functionalization procedures from the literature (i.e. CNM oxidation followed

Table 1. Reactions catalyzed by functionalized carbon nanomaterials.

\begin{tabular}{|c|c|c|c|c|c|}
\hline \multirow{2}{*}{$\begin{array}{l}\text { Type of catalyzed } \\
\text { reaction(s) }\end{array}$} & \multirow{2}{*}{$\begin{array}{l}\text { Covalently tethered } \\
\text { surface functionalities at } \\
\text { 1D-2D CNMs }\end{array}$} & \multicolumn{3}{|c|}{$\begin{array}{c}\text { Dominant surface } \\
\text { properties: }\end{array}$} & \multirow{2}{*}{ Ref. } \\
\hline & & acid & basic & $\begin{array}{l}\text { elec- } \\
\text { tronic }\end{array}$ & \\
\hline \multirow{2}{*}{$\begin{array}{l}\text { Hydrolysis, esteri- } \\
\text { fication and trans- } \\
\text { esterification }\end{array}$} & Aryl sulfonic acids & $\checkmark$ & - & - & {$[9-12]$} \\
\hline & Tertiary aliphatic amines & - & $\checkmark$ & - & {$[13-15]$} \\
\hline \multirow{3}{*}{$\begin{array}{l}\text { Carbon-carbon bond } \\
\text { forming reactions }\end{array}$} & Aryl sulfonic acids & $\checkmark$ & - & - & {$[16]$} \\
\hline & Hydroxyl groups & $\checkmark$ & - & - & {$[17]$} \\
\hline & Aliphatic amines & - & $\checkmark$ & - & {$[18,19]$} \\
\hline \multirow{3}{*}{$\begin{array}{l}\text { Reactions for } \mathrm{CO}_{2} \\
\text { valorization }\end{array}$} & Hydroxyl groups & $\checkmark$ & - & - & {$[20]$} \\
\hline & $\begin{array}{l}\text { Ammonium salts/aliphat- } \\
\text { ic amines/ silanol groups }\end{array}$ & $\checkmark$ & $\checkmark$ & - & {$[21]$} \\
\hline & Pyridine groups & - & $\checkmark$ & - & {$[22]$} \\
\hline \multirow{2}{*}{$\begin{array}{l}\text { Electrochemical } \\
\text { Oxygen Reduction } \\
\text { Reactions (ORR) }\end{array}$} & Ammonium salts & - & - & $\checkmark$ & {$[23]$} \\
\hline & $\mathrm{N}$-containing heterocycles & - & - & $\checkmark$ & $\begin{array}{l}{[19,} \\
24-26]\end{array}$ \\
\hline
\end{tabular}

by carboxylic acid activation with acyl chloride and amine nucleophilic substitution) are known to introduce side-functional groups whose presence is responsible for reduction of the catalyst selectivity (with definitive poisoning in some cases).

\subsection{Carbon-Carbon Bond Forming Reactions}

This class of reactions represents one of the most useful and fundamental transformations at the heart of synthetic organic chemistry. Chemically functionalized nanocarbons in the form of acid and base solid catalysts have been shown to catalyze $\mathrm{C}-\mathrm{C}$ C bond forming reactions, acting as stable and long-term re-usable heterogeneous systems. Poly(styrene sulfonic acid)-grafted carbon nanotubes have successfully been tested as solid catalysts for the alkylation of hydroquinone with tert-butanol.[16] They were prepared by in situ radical polymerization of sodium styrene sulfonate and demonstrated higher activity and good stability in the alkylation reaction with better conversions and selectivity than $-\mathrm{SO}_{3} \mathrm{H}$ decorated samples prepared through direct CNMs sulfonation. In 2011 Song's group reported on $\mathrm{C}_{60}$-fullerene decorated with hydroxyl groups $\left(\mathrm{C}_{60}\right.$-fullerenol) and its exploitation as a valuable catalyst for a series of $\mathrm{C}-\mathrm{C}$ bond forming reactions. ${ }^{[17]}$ The presence of surface hydroxyl groups fosters hydrogen bonding between catalyst and reactants, thus allowing selected $\mathrm{C}-\mathrm{C}$ bond forming reactions to take place. At odds with the poor catalytic performance of the benchmark $\mathrm{Fe}(\mathrm{OH})_{3}, \mathrm{C}_{60}$-fullerenol as organocatalyst has demonstrated high catalytic activity in Henry reactions with remarkable stability and complete selectivity. $\mathrm{C}_{60}$-fullerenol has also promoted aldol condensation, another important reaction involved in many pharmaceutical intermediates and natural products synthesis. Complete selectivity towards $\beta$-hydroxyl ketones was achieved in this reaction. Finally, the same organocatalyst exhibited good to excellent activity in Michael additions as well as Friedel-Crafts reactions. In 2015, the same group prepared amino- 


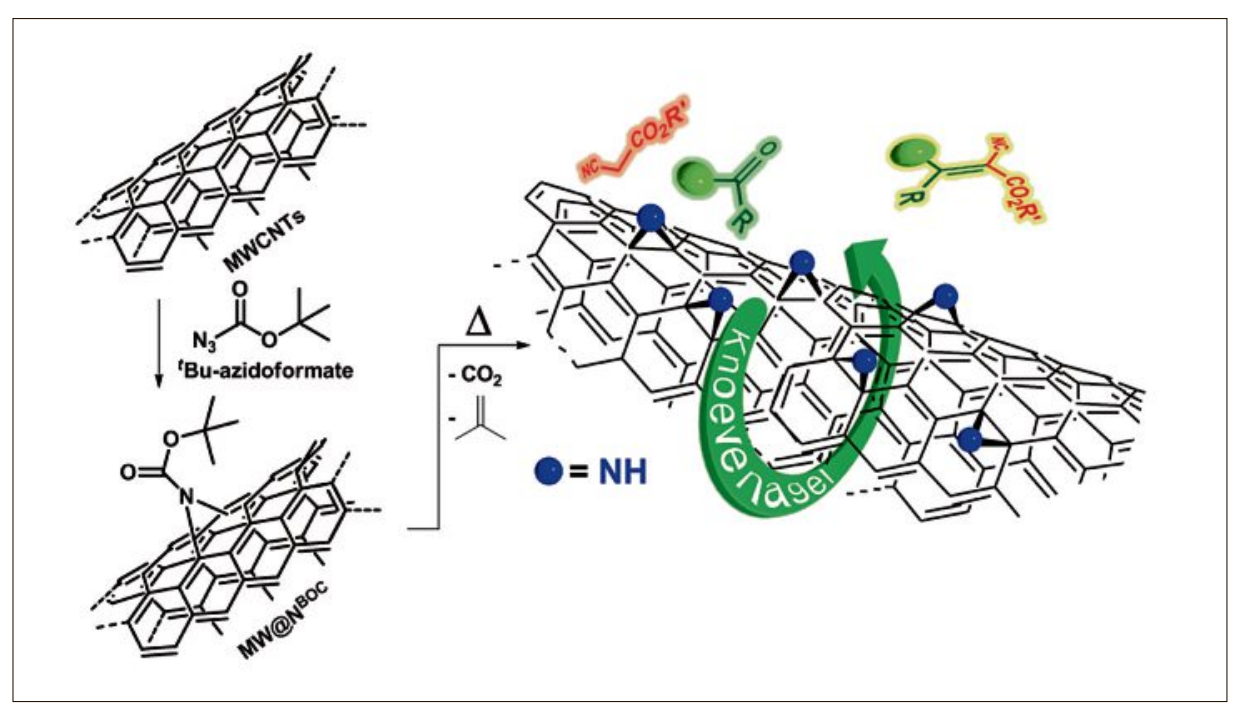

Fig. 2. Sidewall functionalization of MWCNTs by [2+1] cycloaddition followed by controlled thermal decomposition of $\mathbf{M W} @ \mathbf{N}^{B O C}$ to give $\mathbf{M W} @ \mathbf{N}^{\mathbf{A z}}$ as solid base catalyst for the Knoevenagel condensation. Figure loosely reproduced with permission from the American Chemical Society, ref. [19]: ACS Appl. Mater. Interfaces 2016, 8, 30099.

decorated $\mathrm{C}_{60}$-fullerene as solid base catalysts for the Knoevenagel condensation. ${ }^{618]}$ Several aromatic/aliphatic carbonyl compounds and acid carbons as nucleophiles (i.e. ethyl cyanoacetate dimethyl malonate and 2,4-pentanedione) have been successfully scrutinized in the process, showing excellent catalyst performance and tolerance towards different functional groups. Better catalytic performance in the same condensation process has recently been described by Tuci and co-workers using aziridine-functionalized MWCNTs as a heterogeneous and easy-to-reuse catalyst

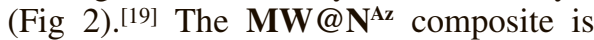
straightforwardly prepared following a two-step synthetic procedure: a $[2+1]$ cycloaddition of tert-butyl azidoformate to the MWCNTs sidewall followed by the controlled thermal decomposition of the carbamate moiety.

As a result, aziridine $\mathrm{NH}$-functionalized materials are obtained. The as-prepared catalyst showed superior activity in the Knoevenagel process with respect to related N-doped and N-decorated carbon nanomaterials of the state-of-the-art. Notably, the aziridine functionalized MWCNTs are easily recovered after each catalytic run and re-used without any apparent loss of activity.

\subsection{Reactions for $\mathrm{CO}_{2}$ Valorization}

Mitigation of the effects caused by $\mathrm{CO}_{2}$ overproduction represents one of the most urgent and challenging issues of modern society. ${ }^{[27,28]}$ In recent years, the scientists' point of view on carbon dioxide has radically changed so that the contemporary chemical community does not regard $\mathrm{CO}_{2}$ as a waste product from fossil fuel but rather as a chemical resource to be harvested and recycled into products of added value with the assistance of a catalyst. ${ }^{[29]}$ One common strategy to take advantage of $\mathrm{CO}_{2}$ is given by its insertion into epoxide derivatives to produce cyclic carbonates, ${ }^{[30,31]}$ very useful as polar aprotic solvents and raw materials for engineering plastics. Since hydrogen bonding between catalyst and epoxide was found to be a key factor for promoting $\mathrm{CO}_{2}$ cycloaddition, $\mathrm{CaO}$ and Song's group has recently exploited the aforementioned $\mathrm{C}_{60}$-fullerenol (see section 2.2) as a heterogeneous metal-free system for the process. ${ }^{[20]}$ It turned out to be an excellent promoter of $\mathrm{CO}_{2}$ cycloaddition to propylene oxide to form propylene carbonate. Later, Yin et al. described a multi-functional graphene oxide (GO) material for the same catalytic reaction. ${ }^{[21]}$ To this aim, the authors have conceived a straightforward strategy for the surface grafting with quaternary ammonium salts. The synthesis consists in the silylation of GO with chlorine-terminated silanes followed by nucleophilic attack by tertiary amines. The as-prepared multi-functional material (containing silanol groups, quaternary ammonium salts and amines) has shown superior catalytic performance in the cycloaddition of $\mathrm{CO}_{2}$ to propylenoxide (PO). The authors claimed that covalently linked amines acted as adsorption sites and activators for $\mathrm{CO}_{2}$, whereas silanol groups provided activation sites for PO stabilizing the ring opened oxirane before the intramolecular $\mathrm{CO}_{2}$ addition and subsequent cyclization step (Fig. 3).

A highly attractive process for $\mathrm{CO}_{2}$ valorization is represented by its catalytic conversion into methanol. This transformation addresses two key issues at one time: a) the reduction of global warming and related climate changes; $b$ ) the production of a synthetic fuel $\left(\mathrm{CH}_{3} \mathrm{OH}\right)$ that can be exploited as alternative energy source. ${ }^{[32,33]}$ Tuci, Giambastiani and co-workers have recently demonstrated the effectiveness of pyridine-decorated MWCNTs ( $\left.\mathbf{N}^{\mathbf{P y}}-\mathbf{M W}\right)$ as the first robust, stable and reusable heterogeneous metal-free catalyst for $\mathrm{CO}_{2}$ reduction with hydroboranes at ambient conditions to afford methoxyborane. ${ }^{[22]}$ With turn-over numbers close to those claimed for other metal and metal-free homogeneous systems of the state-of-the-art and their effective re-use in catalysis, $\mathbf{N}^{\mathbf{P y}} \mathbf{-} \mathbf{M W}$ candidates are the heterogeneous benchmark for this process. Noteworthy, the authors have demonstrated for the first time the key role played by the carbon nanotube carrier as an electronic reservoir for the dangling pyridine active arms engaged in the catalytic cycle. This finding opens a new perspective for the intriguing idea that the CNMs have a non-innocent role on the

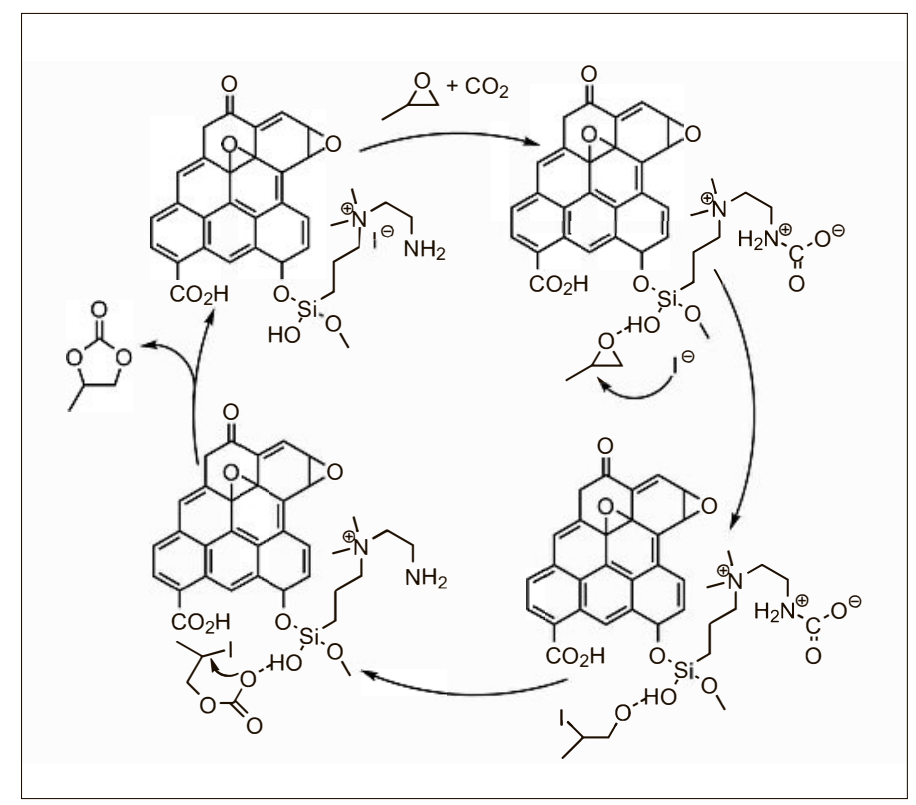

Fig. 3. Supposed reaction mechanism for the $\mathrm{CO}_{2}$ cycloaddition to $\mathrm{PO}$ catalyzed by multifunctional GO. Figure loosely reproduced with permission of Elsevier, ref. [21]: Carbon 2015, 93, 22. 
catalytic activity of the active sites covalently linked to their outer surface.

\subsection{Electrochemical Oxygen Reduction Reaction (ORR)}

Due to their excellent electrical conductivity, carbon-based nanomaterials have emerged in the last decade as highly attractive electrocatalytic systems for a number of key processes at the heart of renewable energy technologies. ${ }^{[34,35]}$ Since the seminal report by Dai and coworkers, ${ }^{[36]}$ many carbon nanomaterials (prevalently in the form of $\mathrm{N}$-doped systems) have been employed as valuable metal-free electrocatalysts for the oxygen reduction reaction (ORR). ${ }^{[37]}$ Despite an impressive number of papers describing $\mathrm{N}$-doped carbon nanomaterials prepared with classical in situ techniques as catalysts for ORR, only a few papers deal with the synthesis of N-decorated CNMs obtained through a tailored chemical functionalization of their outer surface by defined functional groups. The latter approach offers a unique platform for the in-depth understanding of the underlying ORR mechanism. In 2011 Dai and co-workers reported on carbon nanotubes functionalized with poly(diallyldimethylammonium chloride) (PDDA). ${ }^{[23]}$ The quaternary ammonium groups in the polyelectrolyte backbone are claimed to induce a net positive charge on CNTs. The presence of the extra charge makes them efficient metal-free systems for dioxygen activation and its subsequent electrochemical reduction. The as-prepared material has shown remarkable electrocatalytic activity with performance close to that of the benchmark $\mathrm{Pt} / \mathrm{C}$ catalyst with even higher alcohols tolerance and stability under operative conditions. Later, Tuci and coworkers used a radical functionalization protocol (Tour protocol) for the chemical grafting of various $\mathrm{N}$-heterocycles on MWCNTs. The as-prepared composites, featuring 'edge-type $\mathrm{N}$-defect mimics' have been successfully employed as metalfree systems for the same electrochemical process. This alternative approach to the generation of $\mathrm{N}$-decorated nanomaterials offers a unique tool for the comprehension of the role of different $\mathrm{N}$-configurations (pyridinic, pyrrolic, quaternary nitrogens) at the nanomaterial surface, with respect to their ability to activate $\mathrm{O}_{2}$ and make it available for its reduction through a prevalent $4 \mathrm{e}^{-}$process. ${ }^{[24]}$ The same authors have demonstrated the existence of a rational trend (coherent with the Sabatier's principle) between the electronic charge density distribution at the grafted $\mathrm{N}$-heterocycles and their final control on the overpotential value at which the ORR starts.[25,26] This trend can be summarized in a volcano-plot representation for a quick visual identification of the most suitable N-containing groups for ORR catalysis (Fig. 4).

Finally, the same authors found that aziridine-decorated MWCNTs (MW@ $\mathbf{N}^{\mathrm{Az}}$, see section 2.2) are effective metalfree systems for promoting ORR in alkaline environment. ${ }^{[19]}$ Indeed, the covalent insertion of $-\mathrm{NH}$ groups within the outer sidewall of CNTs is claimed to generate a
Fig. 4. Top: MWCNT $\mathrm{N}$-decoration via the aryldiazonium salt (Tour) protocol. Bottom: A volcano plot representation for the 'just right'interaction between $\mathrm{O}_{2}$ and tailored $\mathrm{N}$-groups at the outer surface of chemically decorated MWCNTs engaged in the ORR. net redistribution of the electronic charge density at the N-neighboring carbon sites. Such an electronic effect is thought to foster $\mathrm{O}_{2}$ adsorption and activation, on the way to its ultimate reduction under electrochemical conditions.

\section{Conclusions}

The potential of chemical functionalization for the design and synthesis of tailored hetero-doped CNM-based catalysts has been described. The progress of the functionalization strategies has led to the achievement of fine control of the nanomaterials surface properties. Several composites from the series of ad hoc functionalized CNMs have successfully been employed as heterogeneous metal-free catalysts in a relatively high number of reactions (hydrolysis/esterification/transesterification reactions, $\mathrm{C}-\mathrm{C}$ bond forming reactions, processes for the $\mathrm{CO}_{2}$ valorization and electrochemical reduction of oxygen (ORR)). Noteworthy, in many cases they have shown better performance along with higher stability and long-term durability than benchmark metal-based catalysts of the state-of-the-art or than that of 'randomly' heterodoped CNMs as classically obtained through in situ doping procedures (CVD). Most importantly, chemical protocols for tethering specific chemical entities at the nanomaterial surface offer a unique work basis to shed light on the reaction mechanisms involved in catalytic processes, thus paving the way to the design of more active and selective metal-free heterogeneous catalysts. Selected issues from recent literature have contributed to show the non-innocent role of CNMs on the final catalytic activity of the resulting composites. Such a result adds an additional level of complexity to CNMs in catalysis and moves their role far beyond that of simple carriers for covalently linked active sites. Although many other applications in catalysis still remain to be explored, the tailoring approach to the nanomaterial decoration with well-defined chemical functionalities deserves promising outcomes to the development of active, stable and selective single-phase heterogeneous catalysts and offers unique chances to the comprehension of their intimate mechanism of action.

\section{Acknowledgements}

The Italian MIUR through the PRIN 2015 Project SMARTNESS (2015K7FZLH) 'Solar driven chemistry: new materials for photo- and electro-catalysis' is gratefully acknowledged for financial support. GT also thanks Fondazione 'Ente Cassa di Risparmio di Firenze' for its support to the HORIZON project.

Received: July 28, 2017 
[1] M. Li, F. Xu, H. Li, Y. Wang, Catal. Sci. Technol. 2016, 6, 3670 .

[2] E. Pérez-Mayoral, V. Calvino-Casilda, E. Soriano, Catal. Sci. Technol. 2016, 6, 1265.

[3] X. Liu, L. Dai, Nat. Rev. Mater. 2016, 1, 16064

[4] S. Agnoli, M. Favaro, J. Mater. Chem. A 2016, 4, 5002 .

[5] M. A. Patel, F. Luo, M. R. Khoshi, E. Rabie, Q. Zhang, C. R. Flach, R. Mendelsohn, E. Garfunkel, M. Szostak, H. He, ACS Nano 2016, 10, 2305.

[6] M. Melchionna, S. Marchesan, M. Prato, P. Fornasiero, Catal. Sci. Technol. 2015, 5, 3859.

[7] N. Karousis, N. Tagmatarchis, Chem. Rev. 2010, 110, 5366 .

[8] P. Singh, S. Campidelli, S. Giordani, D. Bonifazi, A. Bianco, M. Prato, Chem. Soc. Rev. 2009, 38, 2214.

[9] X. Wang, R. Liu, M. M. Waje, Z. Chen, Y. Yan, K. N. Bozhilov, P. Feng, Chem. Mater. 2007, 19, 2395.

[10] R. Liu, X. Wang, X. Zhao, P. Feng, Carbon 2008, 46, 1664.

[11] J. Ji, G. Zhang, H. Chen, S. Wang, G. Zhang, F. Zhang, X. Fan, Chem. Sci. 2011, 2, 484.

[12] D. R. Stellwagen, F. van der Klis, D. S. van Es, K. P. de Jong, J. H. Bitter, ChemSusChem 2013, 6,1668 .

[13] J.-P. Tessonnier, A. Villa, O. Majoulet, D. S. Su, R. Schlögl, Angew. Chem. Int. Ed. 2009, 48, 6543.
[14] A. Villa, J.-P. Tessonnier, O. Majoulet, D. S. Su, R. Schlögl, Chem. Commun. 2009, 4405.

[15] A. Villa, J.-P. Tessonnier, O. Majoulet, D. S. Su, R. Schlögl, ChemSusChem 2010, 3, 241.

[16] K. Liu, C. Li, X. Zhang, W. Hua, D. Yang, J. $\mathrm{Hu}, \mathrm{Y}$. Yue, Z. Gao, Catal. Commun. 2010, 12, 217.

[17] F. Niu, J. Wu, L. Zhang, P. Li, J. Zhu, Z. Wu, C. Wang, W. Song, ACS Catal. 2011, 1, 1158.

[18] Y. Sun, C. Cao, P. Huang, S. Yang, W. Song, RSC Adv. 2015, 5, 86082.

[19] G. Tuci, L. Luconi, A. Rossin, E. Berretti, H. $\mathrm{Ba}, \mathrm{M}$. Innocenti, D. Yakhvarov, S. Caporali, C. Pham-Huu, G. Giambastiani, ACS Appl. Mater. Interfaces 2016, 8, 30099.

[20] Y.-B. Sun, C.-Y. Cao, S.-L. Yang, P.-P. Huang, C.-R. Wang, W.-G. Song, Chem. Commun. 2014, 50, 10307.

[21] D.-H. Lan, L. Chen, C.-T. Au, S.-F. Yin, Carbon 2015, 93, 22.

[22] G. Tuci, A. Rossin, L. Luconi, C. Pham-Huu, S. Cicchi, H. Ba, G. Giambastiani, 2017, submitted.

[23] S. Wang, D. Yu, L. Dai, J. Am. Chem. Soc. 2011, 133,5182

[24] G. Tuci, C. Zafferoni, P. D'Ambrosio, S. Caporali, M. Ceppatelli, A. Rossin, T. Tsoufis, M. Innocenti, G. Giambastiani, ACS Catal. 2013, 3, 2108

[25] G. Tuci, C. Zafferoni, A. Rossin, A. Milella, L. Luconi, M. Innocenti, L. Truong Phuoc, C.
Duong-Viet, C. Pham-Huu, G. Giambastiani, Chem. Mater. 2014, 26, 3460.

[26] G. Tuci, C. Zafferoni, A. Rossin, L. Luconi, A. Milella, M. Ceppatelli, M. Innocenti, Y. Liu,

C. Pham-Huu, G. Giambastiani, Catal. Sci. Technol. 2016, 6, 6226.

[27] D. R. Feldman, W. D. Collins, P. J. Gero, M. S. Torn, E. J. Mlawer, T. R. Shippert, Nature 2015 , 519, 339.

[28] M. E. Mann, Proc. Natl. Acad. Sci. USA 2009, 106,4065 .

[29] G. A. Ozin, Energy Environ. Sci. 2015, 8, 1682.

[30] M. North, R. Pasquale, C. Young, Green Chem. 2010, 12, 1514

[31] M. Cokoja, M. E. Wilhelm, M. H. Anthofer, W. A. Herrmann, F. E. Kìhn, ChemSusChem 2015 8, 2436.

[32] W. Wang, S. Wang, X. Ma, J. Gong, Chem. Soc. Rev. 2011, 40, 3703.

[33] M. Aresta, 'Carbon Dioxide as Chemical Feedstock', Wiley-VCH Verlag GmbH \& Co. KGaA, betz-druck GmbH, Darmstadt, 2010.

[34] L. Dai, Y. Xue, L. Qu, H.-J. Choi, J.-B. Baek, Chem. Rev. 2015, 115, 4823.

[35] M. Zhou, H.-L. Wang, S. Guo, Chem. Soc. Rev. 2016, 45,1273 .

[36] K. Gong, F. Du, Z. Xia, M. Durstock, L. Dai, Science 2009, 323, 760 .

[37] K.-H. Wu, D.-W. Wang, D.-S. Su, I. R. Gentle, ChemSusChem 2015, 8, 2772. 